\title{
EDITORIAL
}

\section{CULTURA DE LOS CUIDADOS. UN RECORRIDO DE DIEZ AÑOS}

Elena Ferrer Hernández

Diplomada en Enfermería

Profesora Asociada D epartamento de Enfermería

E.U.E. U niversidad de Alicante

\section{CULTURE OF CARE: TEN YEARS OF LIFE}

\section{SUMMARY}

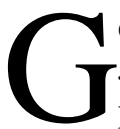
etting to know the CULTURE OF CARE JOURNAL means being acquainted with part of the Nursing History. After 10 years of life, it is a well consolidated reality due basically - from the very beginning - to the effort, determination and hope of a small group of nursing students highly stimulated in their motivation by Professor and Editor, José Siles.

The aim of the present study is to share with the readers the path followed by the CULTURE OF CARE JOURNAL up to its present identity.

To maintain a chronological order a panoramic view in our 'Family Album' is held according to the events that contributed to give the JOURNAL its personal character, reputation and prestige.

Key Words: Culture and Nursing, Nursing History, Anthropology

\section{RESUMEN}

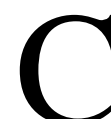

onocer la historia de la revista Cultura de los Cuidados es conocer una parte de la Historia de la Enfermería. Tras 10 años de andadura, es una realidad consolidada gracias, en sus orígenes, a la constancia, empeño, ilusión y esperanza, de un grupo de estudiantes de enfermería, que fueron contagiados por altas dosis de motivación del profesor y director de la revista, José Siles.

Este trabajo tiene el objetivo de compartir con los lectores la trayectoria que ha seguido la revista Cultura de los Cuidados hasta adquirir su identidad.

Para seguir un orden cronológico se relata, oje- ando nuestro Álbum Familiar, los acontecimientos que han contribuido a dar a la revista el sello personal de madurez y prestigio.

Palabras clave: Cultura y enfermería, historia de la enfermería, antropología.

\section{CONTEXO HISTORICO EN EL QUE SURGE CULTURA DE LOS CUIDADOS}

Ni Cultura de los Cuidados ni otra revista de similares características hubiera sido posible sin la existencia previa de otras muchas publicaciones que le precedieron.

En el ámbito internacional hay que destacar la labor de la enfermería estadounidense. La revista de la enfermería norteamericana más antigua fue creada en 1888 (The Trained Nurse and Hospital Review"). Pero la primera revista en la que se publicaron con asiduidad trabajos históricos fue "The american Journal of Nursing" que aparece con el siglo XX, justo un año después de la creación del Consejo Internacional de Enfermería, y que bajo el estímulo de la eminente historiadora de la enfermería, Mary M. Roberts contribuyó al avance de la historia enfermera (DONAHUE, 1988). Sin embargo las revistas de historia de la enfermería y antropología no aparecen hasta la década de los setenta-ochenta del siglo XX y, en general, lo hacen propiciadas por grupos de historiadores de la enfermería que persiguen fines comunes: el mantenimiento de un foro donde poder intercambiar experiencias relativas a sus trabajos históricos, propiciar mecanismos de ayuda para la investigación histórica (becas, premios, congresos), y, en general, contribuir al desarrollo de la historia de la enfermería. 
La Asociación Americana para la Historia de la Enfermería se fundó en 1978 con el nombre original de "Sociedad Internacional de Historia de la Enfermería". Entre sus actividades destacan la publicación de la revista oficial de la asociación con periodicidad anual, la Nursing History Review" el boletín trimestral "Bulletin of the American Association for The history of Nursing".

Otra publicación muy relacionada con la historia de la enfermería, aunque centrada en los estudios antropológicos es la "Revista de Enfermería Transcultural" que aparece a finales de los ochenta también como consecuencia de las necesidades de difusión y vertebración científica de una asociación denominada "Sociedad de Cuidados Transculturales".

Hasta finales de los ochenta, los historiadores de la enfermería españoles no tenían más opción que publicar sus trabajos en revistas de enfermería general. Es a raíz de la fundación de la revista Index de Enfermería cuando se abre la puerta para aquellos que trabajan la historia de la enfermería y desean publicar sus investigaciones en una revista especializada en cuestiones antropológicas e históricas. La primera base de datos nacional de enfermería "Cuiden" surge también al amparo de esta revista y entre sus actividades destaca la organización de cursos de metodología cualitativa y técnicas de documentación.

Híades es la primera revista española exclusivamente dedicada a la historia de la enfermería. Dicha publicación aparece en Agosto de 1994 propiciada por el esfuerzo del Grupo Andaluz para la Investigación de la Historia de la Enfermería (GAIHE).

\section{ORIGEN DE LA REVISTA}

Cultura de los Cuidados, nace tras una gestación a término que estuvo alimentada de entusiasmo, inquietud, dedicación y coraje. Ingredientes todos necesarios para que el nacimiento fuera recibido con gran júbilo.

Los autores de esta creación fueron un grupo de alumnos de Enfermería, unido a la actuación y dirección del profesor José Siles, muy sensibilizado en los temas de Antropología. La iniciativa la lleva Yolanda Gallardo, (Licenciada en el Instituto Nacional de Educación Física). Aprovechando la experiencia de los viajes que realiza a un orfanato de Lima, y tras matricularse en la Diplomatura de Enfermería conoce al grupo de alumnos contagiándole sus inquietudes y aspiraciones.

Este grupo de estudiantes de enfermería crea una ONG Universitaria (con sede en la Escuela de Enfermería), dándose el nombre de Foro de Enfermería para el Desarrollo. Este Foro se crea con el firme propósito de utilizar el ejercicio y la investigación enfermeras para conseguir un mayor equilibrio social, partiendo desde el respeto a las personas y su cultura.

El Foro ha iniciado dos proyectos de Educación para la Salud, uno destinado a niños huérfanos en Perú, y el segundo, en el estudio de la cultura Napuruna (Amazonia peruana). Desde hace dos años no ha habido continuidad en estas actividades.

Paralelamente a esto, el profesor José Siles observa muy de cerca todos los movimientos de los alumnos y envuelto en la misma inquietud y convencido del proyecto se unen todos y propone crear el Seminario de Historia y Antropología de los Cuidados.

Fue una iniciativa propia, pues no se impartía docencia en dicho seminario, se trabajaba en: la realización de artículos, presentación de comunicaciones, desarrollo de proyectos, etc.

Al principio nadie conocía la metodología de investigación y fue el profesor José Siles el que incentivó la formación a los alumnos en esos temas, continuando con el aprendizaje de las otras asignaturas. Asimismo más adelante el profesor Luis Cibanal se incorpora colaborando en el desarrollo del proyecto.

\section{LOS PRIMEROS PASOS}

La trayectoria comienza no estando libre de dificultades que hubo que superar a medida que se iba avanzando. A nivel personal constituyó un reto, fomentado por la ilusión y entusiasmo de todos.

Se dedicaron horas en la lectura de artículos y planificación de actividades. Era un hobby en donde todos los esfuerzos formaban parte de un enriquecimiento personal y profesional.

En ese tiempo el grupo se plantea crear una revista y comienzan a trabajar seleccionando el 
título, el logo y la estructura de la misma. Se unifican los criterios y se aprueba estructurar la revista en cuatro partes básicas: Fenomenología, Historia, Antropología y Teoría y Método.

En agosto del año 1997, en la E.U.E. de la Universidad de Alicante, la revista ve por primera vez la luz.

Cultura de los Cuidados (Revista de Enfermería y Humanidades) es pues una publicación semestral orientada a contribuir al desarrollo del conocimiento histórico, antropológico, fenomenológico y teórico en cuidados enfermeros, constituyendo el conocimiento previo de la culturas el paso preliminar indispensable para cualquier tipo de abordaje investigador en estas materias.

En consecuencia, esta revista surge con la finalidad de servir de soporte de enlace entre la actividad investigadora, especialmente en los campos señalados y las diferentes vertientes que, desarrolladas por profesionales de enfermería en un momento sin duda histórico, están cimentando las bases de un humanismo enfermero.

\section{TRAZANDO CAMINOS}

Convencidos del proyecto fortalecemos nuestros recursos y se plantea formar una Asociación Nacional de Historia y Antropología de los Cuidados Enfermeros.

Se inician los contactos con otros profesionales que están trabajando en los temas de la enfermería transcultural.

Puestos en acción, en Diciembre de 1998, se constituye legalmente, tras la aprobación de los estatutos. Y el día 2 de junio de 1999 se realiza la Presentación en el Paraninfo de la Facultad de Derecho de la Universidad de Alicante. Para tal acto es invitada la Presidenta de la Transcultural Nursing Society, Rachel Spector.

Dicha asociación tiene como fines: promover y desarrollar la investigación histórica y antropológica de los cuidados enfermeros. Servir de plataforma para la contrastación de opiniones, tendencias y estudios históricos y antropológicos en enfermería.

Para el cumplimiento de dichos fines se desarollan actividades como:

La edición de la revista Cultura de los cuidados, organizar seminarios, encuentros, jornadas, colaborar con el Departamento y E.U.E. así como con el Colegio de Enfermería de Alicante y el CECOVA, y con cuantas asociaciones, instituciones y particulares estén interesados en el desarrollo de los mismos fines.

En 1999, se consigue contactar con los compañeros de Transcultural Nursing, enfermeras y enfermeros especializados en el estudio de la enfermería antropológica y transcultural que desde el primer momento con su presidenta a la cabeza Rachel Spector se brindaron a ayudar en el mantenimiento y desarrollo sostenido de nuestro proyecto. A partir de entonces se estrechan las relaciones y se contacta con más gente de la TC (americanas como: Dula Pacquiao, Lancy Purnell, Rick Zoucha, Marty Douglas... ) En los años sucesivos se frecuentan los intercambios y se empiezan la colaboraciones mutuas, con el objetivo de trabajar e investigar la transculturalidad.

Como resultado de estos intercambios se crea un lazo de unión de la Revista Cultura de los Cuidados con el Journal of Transcultural Nursing, siendo nombrado el profesor José Siles miembro del Editorial Board.

La revista queda estructurada en cuatro apartados: Fenomenológica, Historia, Antropología, Teoría y Método. También incluye las secciones: Editorial y Miscelánea.

\section{FENOMENOLOGÍA}

Sección destinada a la publicación de originales y/o revisiones sobre fenomenología clínica. Asimismo tienen cabida en este apartado las experiencias clínicas de los profesionales sea cual sea la forma de expresión: narrativa, ensayo, poética o plástica.

\section{HISTORIA}

Destinada a la publicación de originales y/o revisiones de historia de la enfermería.

\section{ANTROPOLOGÍA}

Sección reservada para la inclusión de originales y/o revisiones de trabajos antropológicos en materia de cuidados enfermeros.

\section{TEORIA Y MÉTODO}

Apartado dedicado a la publicación de originales y/o revisiones que contribuyan tanto al desarro- 
llo teórico-filosófico de la enfermería como a sus métodos y aplicaciones prácticas.

\section{MISCELÁNEA}

Sección dedicada a la divulgación de actividades, noticias, agenda etc., revisión de novedades editoriales.

Al mismo tiempo se han desarrollado acciones tales como la convocatoria anual del premio "Vida y Salud" de Narrativa, convocado por la Escuela Universitaria de Enfermería de Alicante.

En esta convocatoria, se pueden presentar narraciones cuyos contenidos estén relacionados con el ejercicio de la Enfermería o la Fisioterapia. La temática deberá estar relacionada con aspectos que se deriven de estilos de vida saludable, relativa a situaciones reales o ficticias de salud-enfermedad o vinculadas con el mantenimiento y cuidado del medio ambiente.

\section{CONSOLIDANDO POSICIONES}

Con algunos años más de experiencia queremos consolidar nuestros avances y para ello Cultura de los Cuidados se interrelaciona con varias revistas y se ubica en varias bases de datos para situarse en otros horizontes.

Cultura de los Cuidados es Sociofundadora del Consejo de Editores de Revistas de Enfermería Iberoamericanas. Y esta interrelacionada con las revistas: Fundación Index, Pensar Enfermagen y la Revista de Enfermería de la Universidad de Castilla la Mancha en Albacete.

Cultura de los Cuidados esta incluida en varias bases de datos: BDIE, ENFISPO, EBSCO, PUBLISHING, CUIDATGE, CUIDEN, C I N HAL.

La revista la podemos encontrar en formato papel y en soporte informático en la Hemeroteca digital Cantárida.

Cultura de los Cuidados Digital tiene algunas diferencias con su versión impresa:

- Incluye artículos en edición bilingüe, bien porque originalmente se escribieron en otros idiomas, o porque se han traducido expresamente al inglés debido a su interés y para facilitar su difusión internacional.

- Incluye un resumen biográfico de los autores (cuando éstos lo aportan), con el objeto de

facilitar el intercambio científico entre investigadores.

- Incluye un motor de búsqueda automatizada de artículos, que facilita su localización en función de la sección, la materia o el autor.

- Informa sobre los modos de citación de los artículos publicados en la revista, bien en formato digital o impreso.

Contamos con autores de prestigio: profesores de otras Universidades que colaboran en la revista con la publicación de sus artículos: Manuel Amezcua, Francisco Herrera, Carmen de la Cuesta, Juana Hernández, Amparo Nogales.

Cultura de los Cuidados es una publicación seria y sólida de conocimiento humanístico. Constituye un entramado que unifica y apoya las líneas de Enfermería, y en ese sentido se une al proyecto de Doctorado Cultura de los Cuidados.

El 16 de junio de 2004 tuvo lugar la firma del convenio marco de colaboración entre la Universidad de Alicante y la Fundación Index para la realización de actividades de divulgación, de formación y de investigación que redunden en beneficio de ambas entidades, con especial referencia a la promoción de cursos y seminarios de interés mutuo, el desarrollo de investigaciones relacionadas con los cuidados de salud y otras actividades científicas.

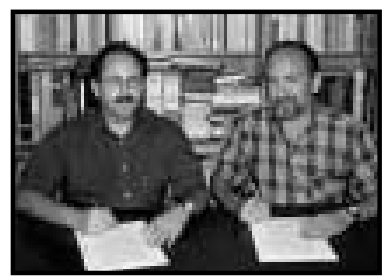

El 16 de junio de 2004, Manuel Amezcua y José Siles firman el convenio marco de colaboración entre la Universidad de Alicante y la Fundación Index

De manera simultánea se procedió a la firma del convenio específico que hace posible la realización del programa de doctorado "Enfermería y Cultura de los Cuidados" en la sede de Index en Granada.

A través de un convenio de colaboración la universidad de Alicante y la Fundación Index convocan el programa de Doctorado "Enfermería y Cultura de los Cuidados" que en la actualidad realiza el Departamento de Enfermería de la citada Universidad. 
El Consejo de Enfermería de la Comunidad Valenciana (CECOVA) forma parte de la revista Cultura de los Cuidados, interviniendo en la gestión administrativa y logística de la misma, siendo su presidente José Antonio Ávila Olivares.

La Financiación de la revista le correspondió en los primeros años al Departamento de Enfermería de la Escuela Universitaria de Enfermería. En la actualidad corre a cargo del CECOVA.

En marzo de 2001, el CECOVA y la Asociación de Historia y Antropología de los Cuidados establecen un marco de colaboración para aunar esfuerzos en la edición y difusión de la revista Cultura de los Cuidados. Dicho acuerdo se concretó en el transcurso de una reunión mantenida entre José Antonio Ávila, presidente del CECOVA y José Siles, director de la revista.

Asimismo esta institución colabora con la organización de las Jornadas anuales que la Asociación Nacional de Historia y Antropología de los Cuidados Enfermeros viene realizando periódicamente.

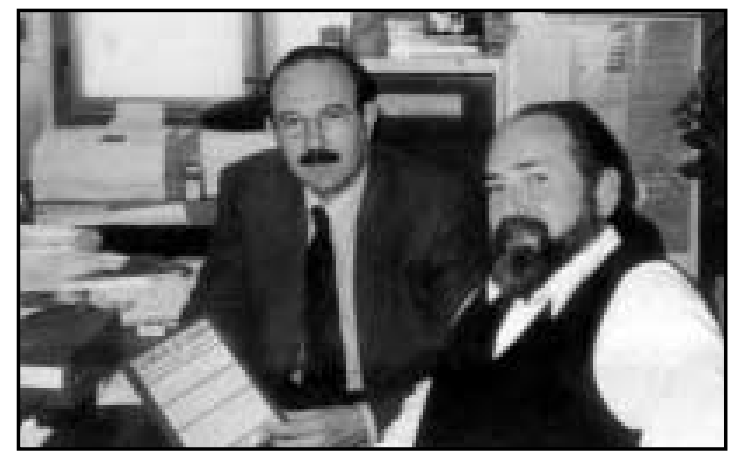

José Antonio Ávila y José Siles plasman el acuerdo de colaboración para potenciar la edición de la Revista

\section{ACTIVIDADES TRANSVERSALES A LA REVISTA}

Como actividades transversales a la revista se encuentran las Jornadas que se han ido organizando y celebrando en años sucesivos.

Las I JORNADAS INTERNACIONALES DE CULTURA DE LOS CUIDADOS se celebraron con fecha 15 y 16 de junio de 2000 en Alicante. Fueron organizadas por la Asociación de Historia y Antropología de los Cuidados y la Escuela de Enfermería de la Universidad de Alicante.
Su temática estuvo polarizada por los cuatro grandes bloques temáticos de la revista: historia, antropología, fenomenología y teoría y método. El nivel internacional de tal evento lo realzaron figuras de la talla de las doctoras: Gordon, Roy y Spector. Los ponentes nacionales fueron: Francisco Herrera, Carmen Chamizo, Manuel Amezcua, los hermanos García Martínez, Ignacio Valle Racero, etc. Todos ellos contribuyeron a desarrollar la enfermería desde sus dimensiones: histórica, antropológica y teórica.

Dos años más tarde se vuelven a celebrar en Alicante las II JORNADAS INTERNACIONALES DE CULTURA DE LOS CUIDADOS. IV REUNIÓN CUALITATIVA EN SALUD, con fecha 14 y 15 de noviembre de 2002 y aquí se presentan seis comunicaciones. Organizado por la E.U.E. Universidad de Alicante con la Asociación Nacional de Historia y Antropología de los Cuidados.

En octubre del año 2002, en Toronto (Canadá) se celebró el CONGRESO MUNDIAL DE ENFERMERÍA TRANSCULTURAL, en donde un grupo de personas de la Asociación de Historia y Antropología de los Cuidados (Manuel Lillo, Isabel Casabona, Roberto Galao, M. Dolores Mora, José Siles, Luis Cibanal y colaboradores) presentaron la comunicación:

"DIFERENCIAS EN LOS SÍNTOMAS MANIFESTADOS POR MUJERES CON ANGINA DE PECHO: UN ESTUDIO INTERNACIONAL DESDE LA PERSPECTIVA DE LA ENFERMERÍA TRANSCULTURAL" .

En el invierno del año 2003, un grupo de la Asociación de Historia y Antropología de los Cuidados visita Estados Unidos para conocer los hospitales Kean University, donde existe un centro de investigación transcultural que dirige la Doctora Dula Pacquiao. Allí se impartieron charlas en un master sobre gestión y enfermería transcultural.

En las III JORNADAS INTERNACIONALES DE CULTURA DE LOS CUIDADOS celebrado en Alicante en junio del 2004, el póster "La influencia de las religiones en la donación de órganos" presentado por Isabel Casabona, Manuel Lillo, $\mathbf{M}^{\mathrm{a}}$ Dolores Mora, Roberto Galao y Purificación Gómez, fue premiado por la Transcultural Nursing Society. 


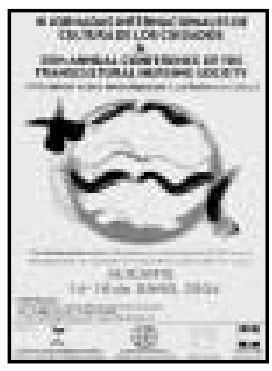

Dicho evento reunió a 230 participantes de 15 países marcándose como objetivos, entre otros, los de debatir la incidencia del género en la educación, la investigación y la práctica en Enfermería transcultural en el mundo, analizar la implicación de la política en los cuidados a inmigrantes y grupos desfavorecidos.

Tanto las perspectivas como los temas o contenidos y los métodos que se abordaron trataron de un único e irrenunciable objetivo, que es el de que mediante el conocimiento se llegue a la tolerancia $\mathrm{y}$ al respeto cultural en toda la disciplina enfermera.

En el año 2005, en New York en la celebración de la Transcultural Nursing Annual Conference, se presentan dos posters:

ASSESSING CULTURALLY COMPETENT CARE AND EVALUATING SATISFACTION FOR DUTCH PATIENTS BELONGING TO THE INTEREUROPEAN PROJECT TO REDUCE WAITING LISTS

A qualitative, transcultural research study in the context of nursing practice

Authors: Manuel Lillo, Isabel Casabona, Roberto Galao, M. Dolores Mora, José Siles, Luis Cibanal.

TRANSCULTURAL NURSING MANEGEMENT IN A CLINICAL CONTEXT

Analysis of Quality perceived by Dutch patients belonging to the Intereuropean Project to reduce waiting lists in Clinica Vistahermosa, Spain

Authors: Isabel Casabona, Manuel Lillo, M. Dolores Mora, Roberto Galao, Luis Cibanal, José Siles.

\section{AVANZANDO}

En estos momentos llevamos 19 revistas editadas. En el período 1997-2006, el número total de artículos publicados ha sido de 215 .

\begin{tabular}{|c|c|c|}
\hline Serciones & Tosad arificulos & Porcensaje \\
\hline Fenomenologia & 44 & 203 \\
\hline Historia & 46 & $21 \%$ \\
\hline Antropologia & 31 & $33 \%$ \\
\hline Tenria y Método & 54 & $25 \%$ \\
\hline
\end{tabular}

Podemos observar como el mayor porcentaje de publicaciones le corresponde al apartado temático "Antropología" (33\%), seguido por Teoría y Método (25\%), Historia (21\%), siendo los trabajos relativos a "Fenomenología" los de menor porcentaje $(20 \%)$.

Dentro de poco cumplimos 10 años, por lo tanto somos jóvenes todavía y es por lo que seguimos creciendo. Hacemos pues, una invitación a investigadores de la teoría, la filosofía y el desarrollo práctico de modelos y métodos enfermeros, la historia de la enfermería, la antropología de los cuidados enfermeros, la fenomenología y a los compañeros y compañeras interesados en publicar sus narraciones, poesías y aquellas otras actividades que destaquen por su carácter humanístico.

La revista Cultura de los Cuidados pretende convertirse en un espacio abierto al debate donde, además del "ejercicio del criterio", se estimule la actividad investigadora y humanística.

La necesidad del conocimiento previo de las culturas como único camino para preservar los niveles mínimos de respeto y dignidad en todo abordaje investigador y/o educativo, revela, por sí misma, la dimensión pragmática del quehacer histórico, antropológico y humanístico en los cuidados enfermeros.

Debemos tener presente el pensamiento que defiende el Informe sobre desarrollo humano 2004, "La libertad cultural en el mundo diverso de hoy". El desarrollo humano es el proceso por el cual se amplían las opciones de la gente para que esta haga y sea lo que valora en la vida. La libertad cultural implica permitir a las personas la libertad de escoger sus identidades y de llevar la vida que valoransin ser excluidas de otras alternativas que les son importantes (como las correspondientes a la educación, la salud o las oportunidades de empleo).

Reconocer las maneras de cuidarse, para vivir saludablemente implica, expresar respeto a los demás aceptando dichas diferencias. En una sociedad plural, como la actual, no todos comparten la misma noción de lo que perjudica o daña. Por tanto, el desafío esta en propiciar, analizando en el contexto, un reencuentro eficaz entre el mundo de la razón o mundo científico y el mundo de la vida. Esto se traduce en saber aprovechar la inmensa riqueza de ideas, valores y símbolos que nos apor- 
tan quienes piensan, viven y se cuidan en forma diferente.

La invitación es pues, a optar por el descubrimiento, por la curiosidad para conocer ese otro ser, con ello, contribuiremos al entendimiento humano y a la apreciación del mundo en toda su grandeza y en todas sus dimensiones.

Fernando Savater nos dice:

"Solo volviendo a la raíz común que nos emparenta podremos los hombres ser cómplices de necesidades que conocemos bien y no extraños encerrados en la fortaleza inasequible de nuestra peculiaridad".

Si el camino se hace andando, hoy queremos continuar en la misma línea en la que un día apostamos y hoy seguimos creciendo, pero resultará del todo imposible seguir caminando en la soledad, por eso, esperamos vuestro apoyo y colaboración, para comprender ese universo de cuidados en nuestro mundo multicultural.

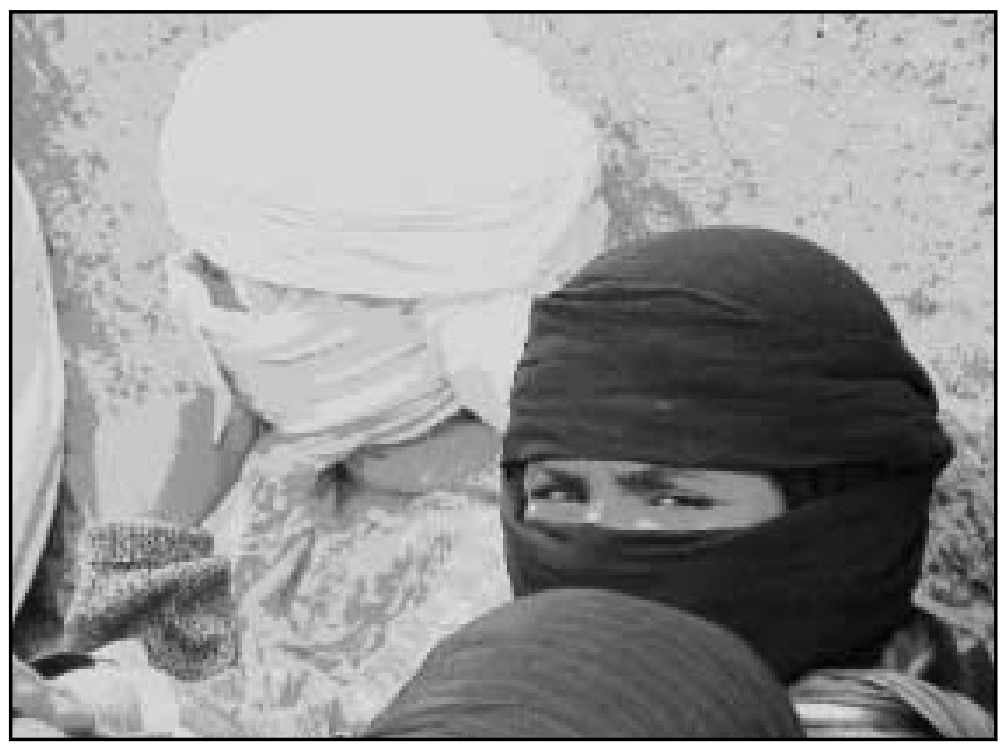

